\title{
Children prioritize humans over animals less than adults do
}

Forthcoming in Psychological Science

\author{
Matti Wilks ${ }^{* 1}$, Lucius Caviola*2, Guy Kahane ${ }^{3}$, Paul Bloom ${ }^{1}$ \\ *Joint first author \\ ${ }^{1}$ Department of Psychology, Yale University \\ ${ }^{2}$ Department of Psychology, Harvard University \\ ${ }^{3}$ Uehiro Centre for Practical Ethics, University of Oxford
}

\begin{abstract}
Author note:
Authors for correspondence: matti.wilks@yale.edu; lucius.caviola@gmail.com
\end{abstract}

All data, analysis, code, and experimental materials are available for download at:

https://osf.io/24ewh/

All authors contributed to the study design and conceptualization. MW collected the data. LC conducted the analyses. All authors contributed to the manuscript preparation.

(C) 2020, SAGE Journals. This paper is not the copy of record and may not exactly replicate the final, authoritative version of the article. Please do not copy or cite without authors' permission. The final article will be available, upon publication, via its DOI:

$10.1177 / 0956797620960398$ 


\begin{abstract}
Is the tendency to morally prioritize humans over animals weaker in children than adults? In two pre-registered studies ( $\mathrm{N}=622)$, 5- to 9-year-old children and adults were presented with moral dilemmas pitting varying numbers of humans against varying numbers of either dogs or pigs and were asked who should be saved. In both studies, children had a weaker tendency to prioritize humans over animals than adults. They often chose to save multiple dogs over one human, and many valued the life of a dog as much as the life of a human. While they valued pigs less, the majority still prioritized ten pigs over one human. By contrast, almost all adults chose to save one human over even one hundred dogs or pigs. Our findings suggest that the common view that humans are far more morally important than animals appears late in development and is likely socially acquired.
\end{abstract}

Keywords: speciesism, moral judgment, development, animals, moral circle 


\section{Statement of relevance}

People everywhere tend to care about and value humans more than nonhuman animals. In two studies, we explored whether this "speciesist" attitude is present even in young children. To find out, we asked 5- to 9-year-olds and adults whether they would choose to save the lives of humans or of dogs and pigs. As expected, most adults were highly "speciesist," choosing to save one human over even one hundred dogs or pigs. But surprisingly, children lacked this pro-human bias. Many children seemed to value the life of a dog as much as the life of a human and chose to save ten pigs over one person. These findings clash with the view held by many philosophers and psychologists, that children have an initially narrow "moral circle" that they gradually expand over development. Instead, they suggest that the perspective that humans are morally special is a socially acquired ideology. It may emerge as children experience the many ways we use animals to serve human needs. 
Almost everyone cares much more about humans than about non-human animals. Across cultures and throughout history, we use animals for food, clothing products, medical experimentation, and entertainment, and we are often indifferent to their suffering.

Psychological research suggests that these common attitudes and practices are linked to the belief that humans matter far more than non-human animals (Amiot \& Bastian, 2017; Caviola et al., 2019; Dhont et al., 2016). For example, in moral dilemmas in which the lives of humans are pitted against the lives of animals, adults consistently prioritize humans over even large numbers of animals (Awad et al., 2018; Petrinovich et al., 1993; Topolski et al., 2013). A recent large-scale study of moral dilemmas involving autonomous cars found that one of the two strongest global preferences is to prioritize saving human lives over those of animals (the other is to save the greater number) (Awad et al., 2018). Another study found that we are less empathic and compassionate towards creatures that are more evolutionarily distant from humans (Miralles et al., 2019).

There are many reasons why people might favor humans over animals. Humans are typically more intelligent, more socially embedded, and are perceived as having a greater capacity to suffer (Caviola et al., 2019). Alternatively, people might prioritize humans over animals simply because of species-membership-they might value humans more merely because they are humans. This is sometimes referred to as speciesism - a term from philosophy that frames our attitude to animals as a prejudice analogous to sexism or racism (Singer, 1975). In support of the speciesism hypothesis, studies have shown that factors such as the lesser mental capacities of animals play only a minor role in explaining our preference for humans (Caviola et al., 2020). For example, in cases where humans with severe cognitive impairment have capacities equivalent to or even lower than some animals, people will nonetheless still prioritize those humans over animals.

The view that humans are morally more important is widespread and shapes law, policy, and behavior. However, the developmental trajectory of this view remains unclear. In this paper, we investigate whether children also prioritize humans over animals and explore potential explanatory mechanisms (e.g. perceived intelligence).

We are not aware of any work that directly compares how children and adults morally prioritize animals relative to humans. Of the few studies that have explored children's attitudes towards animals (see Melson, 2013), only two have systematically 
investigated these intuitions in a developmental context. One study found that 4- to 10-year-old children cared about an increasingly broad range of entities, including animals, as they grew older, but generally cared most for humans (Neldner et al., 2018). Another study tracked how 4 - to 10 -year-old children perceive the moral worth of a range of living, non-living and artificial entities, including a dog and a child (Sommer et al., 2019), and found that children thought it was equally wrong to cause physical harm to a dog and a child, but they also thought that it was more wrong to give away a child than a dog. Thus, while the first study suggests that children might have a tendency to prioritize humans over animals, this second study suggests that their tendency is weaker than in adults.

In order to fully investigate possible age differences in the tendency to prioritize humans over animals we compare children's and adults' responses to moral dilemmas which directly pit humans against dogs and pigs. In order to assess the moral value attributed to humans and animals, these dilemmas contrast varying numbers of humans against varying numbers of either dogs or pigs. Even if participants choose to save a single human over a single dog or pig, for instance, they may still regard the human as just slightly more valuable and hence would not favor a human over two animals.

\section{The present research}

In this project, children and adults were told to consider hypothetical scenarios where two boats and its passengers were sinking and that they had to choose which of the two boats they would rather save (They also had the option of not deciding). More specifically, across several dilemmas, participants were asked whether they would rather save $1,2,10$ or 100 humans or 1, 2, 10, or 100 animals (dogs or pigs).

Based on previous research on adults, we hypothesized that adults would prioritize humans even in cases where many more animals could be saved. By contrast, we hypothesized that children would have a weaker tendency than adults to prioritize humans over these animals. This hypothesis was driven by the findings of a previous study, discussed above, that showed that young children consider harm inflicted on a dog and on a child to be equally wrong (Sommer et al., 2019).

Additionally, we hypothesized that both adults and children have a stronger tendency to prioritize humans over pigs than over dogs, since they value dogs-a 
companion animal-more than pigs—a food animal (Bastian et al., 2012; Bratanova et al., 2011; Loughnan et al., 2010). Finally, in line with past research (Neldner et al., 2018), we hypothesized that children's tendency to prioritize humans over animals increases with age.

\section{Methods}

\section{Open science}

Reports of all measures, manipulations, and exclusions, as well as all data, analysis code, and experimental materials are available for download at https://osf.io/24ewh/.

\section{Ethics statement}

For all studies, relevant ethical guidelines were followed, and the research was approved by the Yale University International Review Board and University of Oxford's Central University Research Ethics Committee.

\section{Study $1 \mathrm{a}$ and $1 \mathrm{~b}$}

This study was pre-registered at https://osf.io/q43zk.

Power Analysis. To obtain $80 \%$ power to detect a small to medium effect $\left(f^{2}=\right.$ .075) with an alpha of .05 in a linear multiple regression with six predictors (animal

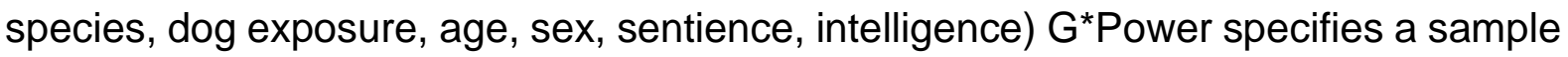
size of 189 . To ensure that we were sufficiently powered, we aimed to collect 220 participants.

We recruited this sample for both adult and child populations. A priori power analysis for a $2 \times 2$ ANOVA with two groups (children vs. adults and two comparisons dogs vs. pigs) revealed that to obtain $80 \%$ power to detect a small-to-medium effect ( $f$ $=.175$ ) with an alpha of .05 , a total sample size of 259 was required. As such, we are sufficiently powered.

Participants. 
Study 1a (Children). We collected a total of 249 participants aged 5-9 yearsi. Participants were tested in a laboratory, a local museum, local schools, public parks and local festivals. The children tested in the laboratory were recruited from a list of parents who had previously agreed to participate. Results did not vary as a function of testing location. An additional 14 participants participated in the experiment but were not included: six due to experimenter error, two due to revoked consent, one due to inattention, and five because they were outside our predefined age rage. $\mathrm{A}$ further 28 were excluded because they failed the two comprehension check questions (plate and worms), leaving us with a final sample of 207 (89 female, $M_{a g e}=$ $7.71, S D_{\text {age }}=1.36$ ). Of these, parents of 117 children opted to report their ethnicities (82\% White/Caucasian; 8\% Black/African American; 4\% Asian; 2\% Indian; 1\% Hispanic; $5 \%$ reported mixed or multiple ethnicities). We also conducted all analyses without any exclusions $(\mathrm{N}=240)$ and when excluding all participants who failed only one comprehension check question $(\mathrm{N}=169)$. All key findings remained the same (see supplementary materials: https://osf.io/24ewh/.

Study $1 b$ (Adults). We recruited 224 US American participants online via MTurk. They received forty cents in payment (in line with US minimum wage) for their participation. Two were excluded for either failing the online attention check, or the two comprehension checks, leaving a final sample of 222 people (93 female, $M_{\text {age }}=37.24$, $\left.S D_{\text {age }}=10.91\right)$. Sample size was determined by the same power analysis employed in the children experiment. Participants reported to have the following ethnicities (multiple selections were possible): 83\% White/Caucasian; 8\% Black/African American; 5\% Asian; 1\% Indian; 7\% Hispanic; 2\% other. Mean religiosity level was 2.44 ( $S D=2.08$ ) on a scale from 1 (Not at all religious) to 7 (Extremely religious). $55 \%$ reported to have no religious affiliation, $32 \%$ reported to be Christians, and the remaining fraction reported to have another religion or belief. The mean political ideology score was $3.30(S D=1.78)$ on a scale from 1 (Very liberal) to 4 (Moderate) to 7 (Very conservative).

Materials and Procedure. The experiment employed a within-subjects design such that all participants saw all questions.

\footnotetext{
i We aimed to collect 220 children. However, because we collected data in group settings (museums, festivals) we ultimately ended up collecting an additional 29 participants. We opted to retain these to account for potential exclusion and to avoid wasting viable data.
} 
Study 1a. Trained lab assistants collected the data. The survey was hosted on Qualtrics and all stimuli were presented on 10.2-inch iPads. A full copy of the survey, including all experimenter scripts, is provided in the supplementary materials. After written parental consent was obtained, participants were given the instructions for the study. They were told that two boats were sinking and that no one on either of the boats is able to swim, but that they could choose to save one boat. They were also told that if it was too hard to choose, they could pick a third option "can't decide".

They subsequently completed a familiarization task (one bike vs. ten pens) with the same response options as the main task (save one bike, save ten pens, can't decide). This was designed to ensure that children understood the conditions of the task. Once they had made their choice, the implications of their decision were explained to them ("you will save one bike, but you won't save ten pens") and they were given the opportunity to change their response as many times as they liked.

After completing the familiarization task, participants completed the main comparisons. These were 18 comparisons of the same structure as the familiarization phase. These comparisons comprised three blocks. One block contained seven human vs. dog questions, another contained seven human vs. pig questions and the third contained four additional questions. The seven questions consisted of 1 human vs. 1 dog/pig ( 2 dogs/pigs, 10 dogs/pigs, 100 dogs/pigs) and 1 dog/pig vs. 1 human (2 humans, 10 humans, 100 humans). The third block consisted of the following control questions: 1 human vs. 10 humans, 1 human vs. 10 worms, 1 human vs. 10 plates, 1 dog vs. 1 pig. The purpose of these control questions was to rule out the possibility that children might merely select the larger number of entities, rather than engaging maturely with the dilemmas.

The entity type and quantity varied for each comparison. The block presentation order, question presentation and comparison side (left vs. right) were all fully randomized. Note that in the studies the term 'person' (or 'people') was used instead of 'human' because we assumed this was easier for children to understand.

After completing main comparisons, participants then completed capacity ratings for all three entities (human, dog, pig). All questions were presented in a randomized order, and the entities were randomized within each question type. The questions were: 1) How smart is a $[x]$ ? 2) How much can [x] feel physical pain? 3) How much can $[x]$ feel sad and scared? Responses were on a scale of 1-4 (not at all, a little bit, a medium amount, a lot). Note that for the analysis we averaged perceived capacity 
to experience physical pain and negative emotions to form a single 'sentience' score. After completion of the capacity ratings, parents were debriefed, and children were offered a prize as a thank you for participating (a small toy). Children tested in schools were not offered a reward.

Justification of stimuli. This experiment involves providing children and adults with "tragic trade-off" dilemmas (Tetlock, 2003). This differs from past work, such as Neldner et al. (2018), which asks children how much they care about different entities. However, caring and moral status are distinct from each other. Here we are interested in moral status attribution, which is closely linked to preventing death and suffering. It is plausible, for example, that someone might dislike people and love animals, but still feel uncomfortable choosing to save a human over an animal. As such, we felt that trade-off dilemmas were best able to capture people's intuitions about the moral status of different beings. Another reason is that trade-off dilemmas require people to directly compare between two options, which allows us to more precisely measure how many animals people think are worth one human. This sort of dilemma captures many reallife zero-sum situations that we face as individuals or society (e.g. where to direct our limited resources). By contrast, the independent ratings used in past work (Neldner et al., 2018) allow participants assign high moral worth to all entities.

We chose dogs because they are a highly valued animal (see Neldner et al., 2018) and would provide a strong test of children's speciesist tendencies. We chose pigs because they are comparable to dogs in many ways (size, behavior, intelligence) but are categorized as a food animal and generally granted less moral status (Caviola \& Capraro, 2020).

We purposefully chose abstract categories of individuals ("human”, "dog”, "pig”), following the standard practice in this sort of research. (Crimston et al., 2016; Greene et al., 2001; Hester \& Gray, 2020; Neldner et al., 2018; Schein, 2020). Future research could investigate the possible effects of more specific characterizations. It is possible that both adults and children would respond differently if the individuals were described in more concrete terms. Research into "identifiable victim" effects (Kogut \& Ritov, 2011), for example, suggests that we value individuals more if they are given names. It is possible that such an effect would be stronger for humans than for dogs or pigs, and hence might lead children to behave more similarly to adults, valuing humans more. Further, we would expect participants to be sensitive to historical and social information about the individuals in question. Many adults, we suspect, would rather 
save a puppy than save a boat with ten serial killers on it. A lot of children would probably save a boat with their mother on it than a boat with any number of animals on it. Further, it's possible that children would prioritize humans over animals more if the humans at stake were children as well, either because they perceive them as peers or as more vulnerable than adults (cf. Goodwin \& Landy, 2013).

Study 1b. This experiment was almost identical to Study 1a, with the only difference that adult participants completed the task online and read the task themselves, rather than having an experimenter administer the task. Adults also indicated their income, education and political orientation.

\section{Study $2 a$ and $\mathbf{2 b}$}

This study was pre-registered at https://osf.io/8twbs.

Power Analysis. To obtain $80 \%$ power to detect a medium effect ( $f=.28$ ) with an alpha of .05, df of 1 and 2 groups (children and adults), a total sample size of 103 was required (i.e., 52 per group). Since we did not plan to conduct a regression analysis as in Study 1, the required sample size was much smaller. In order to account for exclusions, we aimed to recruit 65 participants per group.

\section{Participants.}

Study 2a (Children). We collected a total of 83 participants aged 7-9 years. Six were excluded due to experimenter error, two due to technical issues, one due to parental interference, nine because they were outside our age range, and four because they failed the two comprehension check questions (plate and worms), leaving us with a final sample of 61 (31 female, $M_{a g e}=7.89, S D_{a g e}=0.82$ ). Of these, parents of 30 children opted to report their ethnicity (66\% White/Caucasian; 13\% Black/African American; 1\% Asian; 0.3\% Hispanic; and 0.6\% reported mixed or multiple ethnicities. Children were again tested and recruited by trained research assistants in a dedicated testing lab, at a local museum, local schools and public parks. Again, testing location did not influence results.

Study 2b (Adults). We recruited 66 US American participants online via MTurk. They received thirty-six cents in payment (in line with US minimum wage) for their participation. Two were excluded for either failing the online attention check, or the two comprehension checks, leaving a final sample of 64 people (23 female, Mage $=35$, $S D_{\text {age }}=1.90$ ). Participants reported to have the following ethnicities (multiple 
selections were possible): 88\% White/Caucasian; 8\% Black/African American; 6\% Asian; 2\% Indian; 2\% Hispanic; 0\% other. Mean religiosity level was 2.27 (SD = 1.86) on a scale from 1 (Not at all religious) to 7 (Extremely religious). 63\% reported to have no religious affiliation, $31 \%$ reported to be Christians, and the remaining fraction reported to have another religion or belief. The mean political ideology score was 2.86 $(S D=1.88)$ on a scale from 1 (Very liberal) to 4 (Moderate) to 7 (Very conservative).

Materials and Procedure. We had the same materials and procedure as in Study 1 with two exceptions. First, instead of being asked about their own preferences, participants were introduced to a character "Mr. X", who always does the right thing. They were then asked to report which boat they thought Mr. X would save in each scenario.

Second, we omitted the mental capacity questions. We did this for two reasons. First, because we were interested in determining whether the overall finding was representative of preferences or moral judgements, and thus we were not focused on predicting attitudes. Second, with the smaller sample for the second study we would have been insufficiently powered to accurately identify such predictors.

\section{Analyses}

For the statistical analyses we calculated two scores per participant: a humans-overdogs bias score and a humans-over-pigs bias score. The scores were calculated as follows: each participant received certain points for each dilemma depending on their choice; these points were aggregated per participant. The point scoring system was based on the function $\log _{2}(2 x)$ where $x$ stands for the larger number of beings of the respective dilemma. This would ensure that the scores were weighted by the numbers of beings at stake in the dilemma, but not so much that the dilemmas involving higher number of beings completely dominated the score. For example, it meant that prioritizing one person over 100 dogs contributed more to the humans-over-dogs score than prioritizing one human over 10 dogs, but not ten times more. The maximum score (absolute prioritization of humans) was 14.96 and the minimum score -14.96. A score of zero meant the participants attributed the same moral status to both types of beings. See supplementary materials for more details about the scoring system. 


\section{Results}

\section{Study $1 \mathrm{a}$ and $1 \mathrm{~b}$}

In this study, we presented both children and adults with the same set of moral prioritization dilemmas. We found that children had a much weaker tendency to prioritize humans over animals than adults did (Figure 1). For example, while $71 \%$ of children prioritized 100 dogs over 1 human, 61\% of adults prioritized 1 human over 100 dogs. The 1 vs. 1 dilemmas were particularly revealing: 35\% of children prioritized one human over one dog, $28 \%$ of children prioritized one dog over one human, and the rest couldn't decide. In contrast, $85 \%$ of adults prioritized one human over one dog and only $8 \%$ prioritized the dog. $18 \%$ of children prioritized one pig over one human, $57 \%$ prioritized one human over one pig, and the rest couldn't decide. In contrast, 93\% of adults prioritized one human over one pig and only $3 \%$ prioritized the pig.
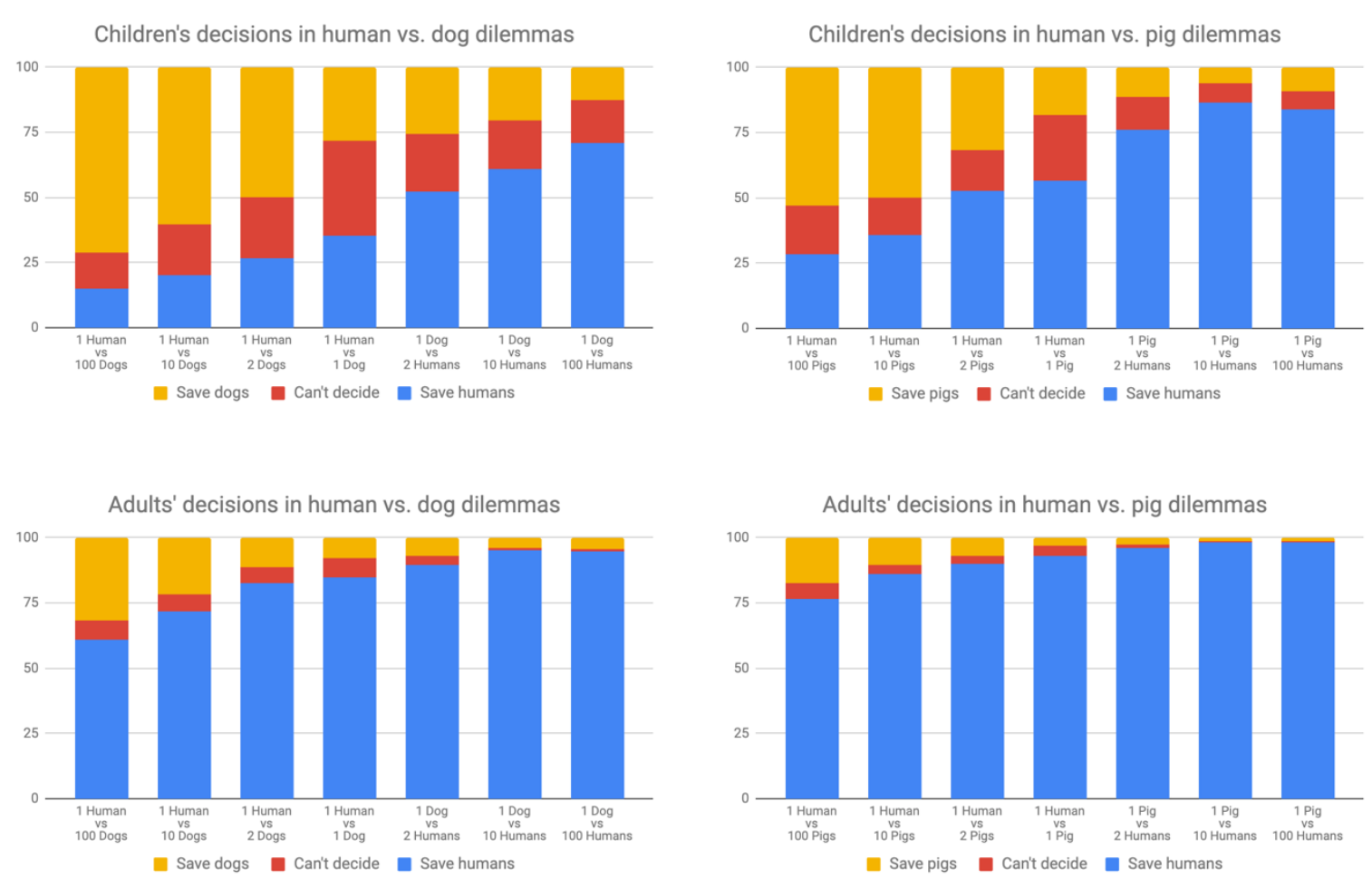

Figure 1. Percentages of either children or adult participants who prioritized the humans, the animals (dogs or pigs), or couldn't decide. (Study 1)

Based on the responses across the seven dilemmas, we calculated a humansover-dogs and humans-over-pigs bias score for each participant. The higher the score, 
the stronger the tendency to prioritize the human over the respective animal species. Children had a mean humans-over-dogs bias score of $0.24(S D=6.66)$ and a humansover-pigs bias score of $4.58(S D=6.41)$. Adults had a mean humans-over-dogs bias score of $9.89(S D=7.47)$ and a humans-over-pigs score of $12.3(S D=5.43)$. A onesample t-test showed that children's humans-over-dogs bias score was not statistically higher than zero, $t(206)=0.52, p=.60, d=.04,95 \% \mathrm{Cl}[-.10, .17]$, suggesting that, on average, children tend not to prioritize humans over dogs. Children's humans-overpigs bias score $(t(206)=10.26, p<.001, d=.71,95 \% \mathrm{Cl}[.56, .87])$ as well as both adults' humans-over-dogs bias score $(t(222)=19.77, p<.001, d=1.32,95 \% \mathrm{Cl}[1.15$, 1.52]) and humans-over-pigs bias score $(t(222)=33.82, p<.001, d=2.26,95 \% \mathrm{Cl}$ $[2.05,2.56])$ were all statistically above zero, suggesting that children do prioritize humans over pigs and adults prioritize humans over both dogs and pigs.

A mixed ANOVA 2 (group: children vs. adults) x 2 (species: pig vs. dog) revealed 2 main effects and an interaction. Children had a weaker tendency to prioritize humans over animals than adults, $F(1,426)=239.03, p<.001, \eta_{p}^{2}=.36,95 \% \mathrm{Cl}[.29, .42]$. Both children and adults had a stronger tendency to prioritize humans over pigs than over dogs, $F(1,428)=143.51, p<.001, \eta_{p}^{2}=.26,95 \% \mathrm{Cl}[.19, .32]$. The difference in bias scores was greater for children than for adults, $F(1,428)=11.85, p<.001, \eta_{p}^{2}$ $=.03,95 \% \mathrm{Cl}[.01, .06]$. This interaction effect, however, could simply be the result of there being a ceiling effect in adults, i.e., their scores were close to the maximum score for both pigs and dogs.

Figure 2 shows that children and adults had similar perceptions about the intelligence and sentience levels of humans, dogs and pigs. They both perceived humans to be more intelligent than dogs and dogs to be more intelligent than pigs. Similarly, they both perceived humans to be more sentient than dogs and dogs to be more sentient than pigs. Notably, the differences in perceived intelligence and sentience for these beings were of similar degree in adults and children. Yet, despite this, children and adults gave different moral judgements, suggesting that perceived intelligence and sentience does not fully account for moral judgments. 

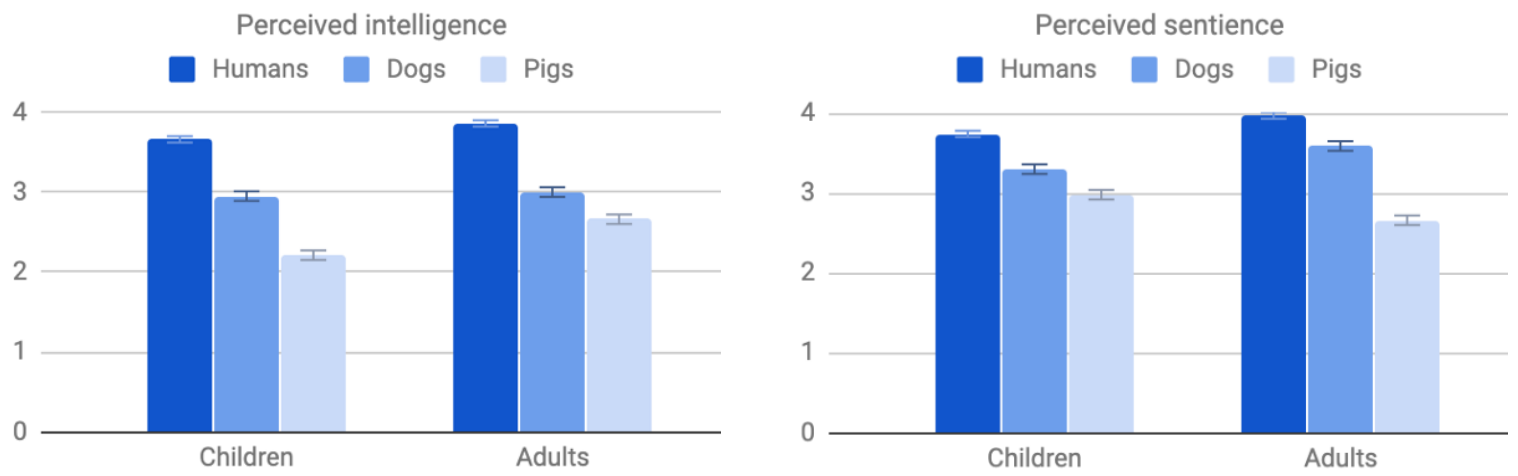

Figure 2. Children and adults' mean ratings of intelligence (1) and sentience (2) for humans, dogs, and pigs. Error bars represent standard errors.

To explore potential explanatory mechanisms, we conducted a linear regression using the bias scores as an outcome variable and with species (dog vs. pig), age, gender, perceived intelligence, perceived sentience, and regular dog (pet) exposure as predictor variables. This was conducted separately for the child and adult sample. Note that we subtracted perceived intelligence and sentience score for the animals from the perceived intelligence and sentience scores of humans for each participant, producing a perceived difference in intelligence/sentience score. The results showed that species, perceived intelligence and dog exposure were significant predictors in both the children and adult sample (Table 1), while gender and perceived sentience were not. Age was predictive in the adult sample, but not the child sample.

Against our hypothesis, there was neither a significant correlation between age and humans-over-dogs bias scores $(r=.12, p=.07)$ nor between age and humansover-pigs bias score $(r=.04, p=.59)$ for children. In adults, however, age correlated positively with humans-over-dogs bias scores $(r=.17, p=.01)$ but not with humansover-pigs bias scores $(r=.09, p=.21)$.

Children who had regular exposure to dogs (45\%) had a lower bias in favor of humans over dogs $(M=-2.26, S D=6.23)$ than children without exposure $(M=2.40$, $S D=6.37), t(198)=5.28, p<.001, d=.74,95 \% \mathrm{Cl}[.45,1.03]$. Similarly, children with dog exposure had a lower bias in favor of humans over pigs $(M=3.02, S D=6.52)$ than children without exposure $(M=5.95, S D=6.10), t(192)=3.29, p=.001, d=.47$, $95 \% \mathrm{Cl}[.18, .75]$. Adults who had regular exposure to dogs (69\%) also had a lower bias in favor of humans over dogs $(M=9.10, S D=7.93)$ than adults without exposure $(M=11.83, S D=5.86), t(173)=2.86, p=.005, d=.37,95 \% \mathrm{Cl}[.08, .66]$. Adults with dog exposure did not have a significantly lower bias in favor of humans over pigs $(M$ 
$=11.83, S D=5.86)$ than adults without exposure $(M=13.14, S D=4.16), t(178)=$ $1.65, p=.10, d=.21,95 \% \mathrm{Cl}[-.08, .50]$.

We found that the pattern of results remains even when we excluded children who seemed to merely pick larger numbers in control questions (e.g., children who picked 10 plates over 1 human). This suggests that the effect cannot be explained merely as a bias in children for choosing the larger number. Moreover, children had a much weaker tendency to prioritize humans over animals than adults even in dilemmas that pitted one human against one dog or pig, i.e., when the numbers on both sides were the same. This further supports the hypothesis that children have a weaker tendency to prioritize humans over animals than adults.

Table 1. Linear regression predicting bias scores

\begin{tabular}{lcc}
\hline & Children & Adults \\
\cline { 2 - 3 } Species & $49.13^{* * *}$ & $15.96^{* * *}$ \\
Age & 1.02 & $5.31^{*}$ \\
Gender & 1.00 & 0.94 \\
Perceived Intelligence & $9.52^{* *}$ & $26.19^{* * *}$ \\
Perceived Sentience & 1.96 & $3.43^{\dagger}$ \\
Regular dog (pet) & $19.15^{* * *}$ & $3.92^{*}$ \\
exposure & & \\
\hline Note. F values. ${ }^{\dagger} p<.10 .{ }^{*} p<.05 .{ }^{* *} p<.01 .{ }^{* * *} p<.001$.
\end{tabular}

\section{Study $2 a$ and $2 b$}

This study aimed to replicate the effects found in the first study. However, instead of asking participants how they personally would decide in the moral dilemmas, we asked them how they think a person who always does the morally right thing would decide. This would allow us to rule out the possibility that children's responses in the first study represented their personal preferences, rather than their views about what is the morally right decision. 

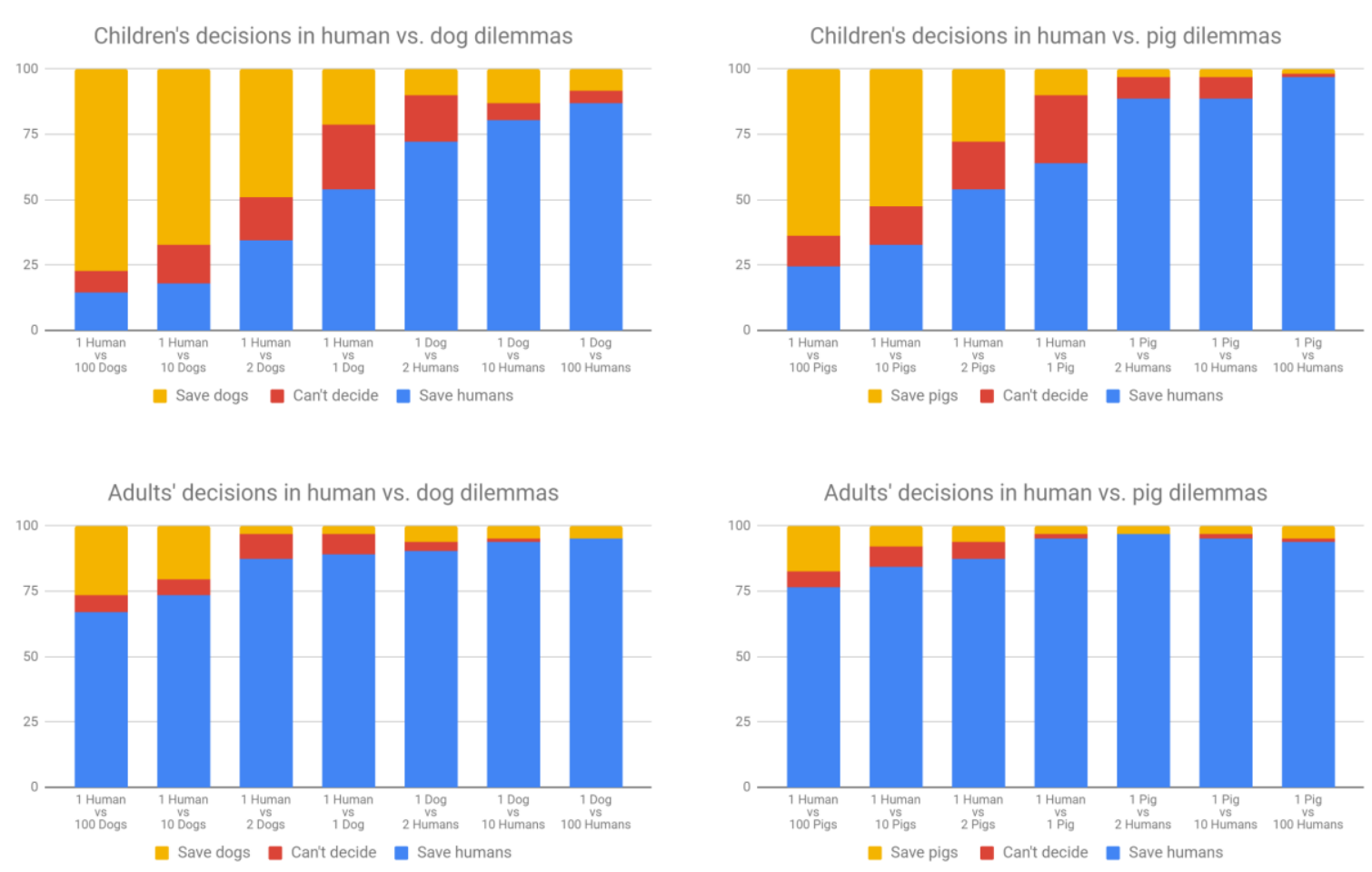

Figure 3. Percentages of either children or adult participants who prioritized the humans, the animals (dogs or pigs), or couldn't decide. (Study 2)

The pattern of results was similar to that of Study 1 (Figure 3). Children had a mean humans-over-dogs bias score of $1.74(S D=6.16)$ and a humans-over-pigs bias score of $5.15(S D=5.65)$. Adults had a mean humans-over-dogs bias score of 10.66 $(S D=6.47)$ and a humans-over-pigs score of $12.02(S D=5.10)$. One-sample t-tests showed that children's humans-over-dogs bias score $(t(60)=2.20, p=.03, d=.28$, $95 \% \mathrm{Cl}[.03, .54]$ as well as their humans-over-pigs bias score $(t(60)=7.13, p<.001$, $d=.91,95 \% \mathrm{Cl}[.61,1.21])$ were significantly above zero. The same was the case for adults' humans-over-dogs bias score $(t(63)=13.19, p<.001, d=1.65,95 \% \mathrm{Cl}[1.27$, $2.03])$ and adults' humans-over-pigs bias score $(t(63)=18.85, p<.001, d=2.36,95 \%$ $\mathrm{Cl}[1.87,2.84])$. This suggests that both children and adults tended to prioritize humans over dogs and pigs.

We found that children had a slightly weaker tendency to prioritize humans over animals in Study 1 than in Study 2. It is possible that this tendency is slightly more reflected in their personal preferences (Study 1) than in their beliefs about what is morally right (Study 2) or what they think adults think is right. Note, however, that the 
discrepancy was minimal and may merely be fortuitous, given the relatively small sample size of Study 2.

A mixed ANOVA 2 (group: children vs. adults) x 2 (species: pig vs. dog) revealed 2 main effects and no interaction. Children had a weaker tendency to prioritize humans over animals than adults, $F(1,121)=72.74, p<.001, \mathrm{np}^{2}=.37,95 \% \mathrm{Cl}[.24, .48]$. Both children and adults had a stronger tendency to prioritize humans over pigs than over dogs, $F(1,124)=23.25, p<.001, \eta_{\mathrm{p}}^{2}=.16,95 \% \mathrm{Cl}[.05, .27]$. There was an interaction effect between group and species, $F(1,124)=4.42, p=.04, \eta_{p}^{2}=.05,95 \% \mathrm{Cl}[.0$, .11].

Again, there was neither a significant correlation between age and humans-overdogs bias scores $(r=.09, p=.47)$ nor between age and humans-over-pigs bias score $(r=-.07, p=.60)$ for children. In adults, however, age correlated positively with humans-over-dogs bias scores $(r=.36, p=.003)$ and with humans-over-pigs bias scores $(r=.37, p=.003)$.

Children who had regular exposure to dogs (68\%) had a lower (marginally sign.) bias in favor of humans over dogs $(M=0.78, S D=5.80)$ than children without exposure $(M=4.25, S D=6.66), t(26)=1.89, p=.07, d=.57,95 \% \mathrm{Cl}[-.03,1.16]$. However, children with dog exposure did not have a lower bias in favor of humans over pigs $(M=5.53, S D=5.43)$ than children without exposure $(M=4.32, S D=6.39)$, $t(26)=-0.68, p=.50, d=.21,95 \% \mathrm{Cl}[-.36, .77]$. Adults who had regular exposure to dogs $(55 \%)$ did not have a significantly lower bias in favor of humans over dogs $(M=$ $9.69, S D=7.15)$ than adults without exposure $(M=11.84, S D=5.42), t(62)=1.36, p$ $=.18, d=.33,95 \% \mathrm{Cl}[-.17, .83]$. Similarly, adults with dog exposure did not have a significantly lower bias in favor of humans over pigs $(M=11.61, S D=4.98)$ than adults without such exposure $(M=12.51, S D=2.29), t(58)=0.69, p=.49, d=.17,95 \% \mathrm{Cl}$ $[-.32, .67]$.

\section{Discussion}

To our knowledge, this is the first project to directly and systematically compare the degree to which children and adults prioritize humans over animals in moral dilemmas. Across two studies we found that children between the age of 5 and 9 have a weaker tendency to prioritize humans over dogs and pigs than adults. This is expressed in both their own preferences (Study 1) and in their beliefs about what is 
morally correct (Study 2). In both studies, the majority of children said they would save multiple dogs over one human. And in 1 vs. 1 scenarios, many opted to save the dog, or couldn't decide. Children did tend to prioritize humans over pigs, but this tendency was weaker than that of adults. In contrast to our predictions, however, we did not find any age-related changes in children's judgements: children from 5-9 years all tended to value animals far more than adults did.

We found that regular exposure to dogs had a strong impact on their tendency to prioritize humans. Children with such background valued dogs much more than those without it. In Study 1, but not Study 2, dog exposure also predicted children's tendency to prioritize humans over pigs.

In Study 1, we found that the extent to which participants perceived dogs and pigs as less intelligent predicted the extent to which they prioritized humans. By contrast, levels of sentience did not predict moral judgments. Notably, we found that even though adults and children attributed roughly the same absolute and relative levels of intelligence and sentience to humans, dogs, and pigs, their moral judgments were strikingly different. This suggests that factors other than intelligence or sentience underpin these moral judgments.

Previous studies suggest that adults exhibit speciesism (Caviola et al., 2019). For example, adults prioritize humans even in cases where humans have equal or lower cognitive capacities than animals (Caviola et al., 2020). Thus, one possible explanation of our findings is that children are far less speciesist than adults. While we found that children weakly prioritize humans over dogs and pigs, we do not know whether this is because of speciesism or because of other factors, such as the belief that humans have more sophisticated cognitive capacities or that they experience more happiness over their lifetimes than dogs or pigs do.

What are the origins of this tendency? One possibility is that it is an unlearned preference. For much of human history, animals played a central role in human lifewhether as a threat or as a resource. It therefore seems possible that humans would develop distinctive psychological mechanisms for thinking about animals. Even if there are no specific cognitive adaptations for thinking about animals, it is hardly surprising that humans prefer humans over animals; similar to their preference for tribe members over strangers. Similarly, given that in-group favoritism in human groups (e.g. racism, sexism, minimal groups) tends to emerge as early as preschool years (Buttelmann \& 
Böhm, 2014), one would expect that a basic tendency to prioritize humans over animals also emerges early.

But we would suggest that the much stronger tendency to prioritize humans over animals in adults has a different source, and, given the lack of correlation between age and speciesism in children, one that emerges late in development. Adolescents may learn and internalize the socially-held 'speciesist' notion—or ideology-that humans are morally special and deserve full moral status, while animals do not. While ideas and practices reflecting strong speciesism are widespread, these may not be as salient to young children from urban backgrounds in developed countries, to whom animals are largely presented in a highly positive and anthropomorphized form. Most young children have no direct experience, and often no knowledge, of the practices relating to, e.g., meat production or animal experimentation. It is possible that strong speciesist beliefs emerge only when these practices become more salient, during adolescence (at least in Western cultures). Thus, the strong form of speciesism exhibited in adults may be a socially acquired ideology.

The hypothesis that speciesism is at least partly a socially acquired ideology could also explain why there are different cultural manifestations of speciesism, e.g., in certain cultures people eat dogs while others consider cows holy. It could also explain why in our studies older adults had a stronger tendency to prioritize humans over animals than younger adults. This could be a reflection of a generational shift in attitudes to animal welfare, perhaps partly influenced by the animal rights movement that emerged in the 1970s (Singer, 1975). While today many people are opposed to unnecessary animal cruelty (Vaughn et al., 2009), historically that was not always the norm (Kelch, 2012; Pinker, 2011)

Further research is required to explore the origins of speciesism. Such research could explore when attitudes shift in adolescence from a weak to a strong tendency to prioritize humans over animals. Or whether the strong form of speciesism exhibited in adults emerges earlier in cultures where younger children have more direct exposure to instrumental uses of animals.

Our study provides initial evidence that children prioritize humans less over animals than adults do. However, there are limits to the generalizability of our findings. One is that, for reasons given earlier, we chose to look at dogs and pigs. People may respond differently when asked about different animals, such as smaller or scarier ones, or those that are more human-like, such as chimpanzees. Similarly, our subjects 
may have responded differently if the individuals at stake were specified in more detail. Another limitation is that our sample was primarily Caucasian, English-speaking and recruited from urban areas. Given the apparent social learning mechanisms, it is possible that children from different backgrounds may respond differently. Another limitation is the use of purely hypothetical dilemmas, rather than real life choices. More research is needed to test the extent to which our finding generalizes across participant populations, moral contexts, and types of beings at stake in both experimental tasks and in real-life behavior.

To sum up, our research suggests that young children are far less speciesist than adults, at least in the context of dogs and pigs. Across two studies, children as old as 9 years prioritized humans to a far lesser extent than adults did, who almost always chose to save humans. This indicates that speciesism may emerge late in development. This challenges the notion that the tendency to morally prioritize humans is a completely ingrained moral intuition, unrelated to social norms. Instead, our findings suggest that, while a general bias in favor of humans may be present in young children, the strong speciesist view held by adults may be socially acquired and, thus, potentially malleable. It is possible, in particular, that strong speciesism would not be as pervasive in a cultural context where, for example, vegetarianism is much more common and direct, positive exposure to animals is more common in adolescence. 


\section{References}

Amiot, C. E., \& Bastian, B. (2017). Solidarity with Animals: Assessing a Relevant Dimension of Social Identification with Animals. PLOS ONE, 12(1), e0168184. https://doi.org/10.1371/journal.pone.0168184

Awad, E., Dsouza, S., Kim, R., Schulz, J., Henrich, J., Shariff, A., Bonnefon, J.-F., \& Rahwan, I. (2018). The Moral Machine experiment. Nature, 563(7729), 59-64. https://doi.org/10.1038/s41586-018-0637-6

Bastian, B., Loughnan, S., Haslam, N., \& Radke, H. R. M. (2012). Don't mind meat? The denial of mind to animals used for human consumption. Personality and Social Psychology Bulletin, 38(2), 247-256. https://doi.org/10.1177/0146167211424291

Bratanova, B., Loughnan, S., \& Bastian, B. (2011). The effect of categorization as food on the perceived moral standing of animals. Appetite, 57(1), 193-196. https://doi.org/10.1016/j.appet.2011.04.020

Buttelmann, D., \& Böhm, R. (2014). The Ontogeny of the Motivation That Underlies In-Group Bias. Psychological Science, 25(4), 921-927. https://doi.org/10.1177/0956797613516802

Caviola, L., \& Capraro, V. (2020). Liking but Devaluing Animals: Emotional and Deliberative Paths to Speciesism. https://doi.org/10.31234/osf.io/sx5uw

Caviola, L., Everett, J. A. C., \& Faber, N. S. (2019). The moral standing of animals: Towards a psychology of speciesism. Journal of Personality and Social Psychology, 116(6), 1011-1029. https://doi.org/10.1037/pspp0000182

Caviola, L., Schubert, S., Kahane, G., \& Faber, N. S. (under review). Why people prioritize humans over animals: A framework for moral anthropocentrism. 
Crimston, D., Bain, P. G., Hornsey, M. J., \& Bastian, B. (2016). Moral expansiveness: Examining variability in the extension of the moral world. Journal of Personality and Social Psychology, 111(4), 636-653. https://doi.org/10.1037/pspp0000086

Dhont, K., Hodson, G., \& Leite, A. C. (2016). Common Ideological Roots of Speciesism and Generalized Ethnic Prejudice: The Social Dominance Human-Animal Relations Model (SD-HARM). European Journal of Personality, 30(6), 507-522. https://doi.org/10.1002/per.2069

Greene, J. D., Sommerville, R. B., Nystrom, L. E., Darley, J. M., \& Cohen, J. D. (2001). An fMRI Investigation of Emotional Engagement in Moral Judgment. Science, 293(5537), 2105-2108. https://doi.org/10.1126/science.1062872 Hester, N., \& Gray, K. (2020). The Moral Psychology of Raceless, Genderless Strangers. Perspectives on Psychological Science, 15(2), 216-230. https://doi.org/10.1177/1745691619885840

Kelch, T. G. (2012). A SHORT HISTORY OF (MOSTLY) WESTERN ANIMAL LAW: PART I. 19, 40.

Kogut, T., \& Ritov, I. (2011). The identifiable victim effect: Causes and boundary conditions. In The science of giving: Experimental approaches to the study of charity (pp. 133-145). Psychology Press.

Loughnan, S., Haslam, N., \& Bastian, B. (2010). The role of meat consumption in the denial of moral status and mind to meat animals. Appetite, 55(1), 156-159. https://doi.org/10.1016/j.appet.2010.05.043

Melson, G. F. (2013). Children's ideas about the moral standing and social welfare of non-human species. Journal of Sociology, 27. 
Miralles, A., Raymond, M., \& Lecointre, G. (2019). Empathy and compassion toward other species decrease with evolutionary divergence time. Scientific Reports, 9(1), 1-8. https://doi.org/10.1038/s41598-019-56006-9

Neldner, K., Crimston, D., Wilks, M., Redshaw, J., \& Nielsen, M. (2018). The developmental origins of moral concern: An examination of moral boundary decision making throughout childhood. PLOS ONE, 13(5). https://doi.org/10.1371/journal.pone.0197819

Petrinovich, L., O’Neill, P., \& Jorgensen, M. (1993). An empirical study of moral intuitions: Toward an evolutionary ethics. Journal of Personality and Social Psychology, 64(3), 467-478. https://doi.org/10.1037/0022-3514.64.3.467

Pinker, S. (2011). The Better Angels of Our Nature: Why Violence Has Declined. Penguin.

Schein, C. (2020). The Importance of Context in Moral Judgments. Perspectives on Psychological Science, 15(2), 207-215. https://doi.org/10.1177/1745691620904083

Singer, P. (1975). Animal Liberation. Random House.

Sommer, K., Nielsen, M., Draheim, M., Redshaw, J., Vanman, E. J., \& Wilks, M. (2019). Children's perceptions of the moral worth of live agents, robots, and inanimate objects. Journal of Experimental Child Psychology, 187, 104656. https://doi.org/10.1016/j.jecp.2019.06.009

Tetlock, P. E. (2003). Thinking the unthinkable: Sacred values and taboo cognitions. Trends in Cognitive Sciences, 7(7), 320-324. https://doi.org/10.1016/S13646613(03)00135-9

Topolski, R., Weaver, J. N., Martin, Z., \& McCoy, J. (2013). Choosing between the Emotional Dog and the Rational Pal: A Moral Dilemma with a Tail. 
Anthrozoös, 26(2), 253-263.

https://doi.org/10.2752/175303713X13636846944321

Vaughn, M. G., Fu, Q., DeLisi, M., Beaver, K. M., Perron, B. E., Terrell, K., \& Howard, M. O. (2009). Correlates of Cruelty to Animals in the United States:

Results from the National Epidemiologic Survey on Alcohol and Related

Conditions. Journal of Psychiatric Research, 43(15), 1213-1218.

https://doi.org/10.1016/j.jpsychires.2009.04.011 